\title{
Comparison of Two Ratio Estimators Using Auxiliary Information
}

\author{
Bawa, Ibrahim ${ }^{1,}$ Makada, Audu ${ }^{2}$ \\ ${ }^{I}$ Federal Inland Revenue Services, Abuja. \\ ${ }^{2}$ Statistics Department, Niger State Polytechnic Zungeru.
}

\begin{abstract}
This study is conducted to compare two ratio estimators that use auxiliary information. The estimators are Olkin average ratio estimator and Kadilar-Cingi estimator. Efficiencies of the estimators are investigated theoretically and using data from Federal Inland Revenue Service Revenue House Store. The result shows Kadilar - Cingi estimator is more efficient than Olkin estimator.
\end{abstract}

Keywords: Auxiliary variable,Ratio Estimators, Efficiency, Comparison.

\section{Indroduction}

The use of auxiliary variable in survey sampling has old history. The auxiliary variable has been efficiently used by numerous survey statisticians to increase the precision of estimates. Laplace (1820) has used the auxiliary information in estimating the population of France. The use of auxiliary information in allocating probabilities of selection has been demonstrated by Hansen and Hurwitz (1943). The auxiliary information also plays very significant role in number of estimating techniques in survey sampling. Cochran (1942) demonstrated the use of auxiliary information in defining the ratio estimator of population mean and total. Cochran, (1942) also introduced the regression estimator as more efficient method of estimation in survey sampling. Srivastava (1967) had shown the efficient use of auxiliary information in improving the performance of estimator. The use of multiple auxiliary variables in estimation has been introduced by Raj (1965). Several ratio type and chain ratio type estimators have been discussed in the literature using the information on single and multiple auxiliary variables.

The estimation of the population mean is a persistent issue in sampling practice and many efforts have been made to improve the precision of the estimates. The literature on survey sampling describes a great variety of techniques for using auxiliary information by means of ratio, product and regression methods. Particularly, in the presence of multi-auxiliary variables, a wide variety of estimators have been discussed, following different ideas, and linking together ratio, product or regression estimators, each one exploiting the variables one at a time.

Olkin (1958) was the first author to deal with the problem of estimating the mean of a survey variable when auxiliary variables are made available. He suggested the use of information on more than one supplementary characteristic, positively correlated with the study variable, considering a linear combination of ratio estimators based on each auxiliary variable separately. The coefficients of the linear combination were determined so as to minimize the variance of the estimator. Analogously to Olkin, Singh (1967) gave a multivariate expression of Murthy (1964) product estimator, while Raj (1965) suggested a method for using multi-auxiliary variables through a linear combination of single difference estimators. Moreover, Singh (1967) considered the extension of the ratio-cum-product estimators to multi-supplementary variables, while Rao and Mudholkar (1967) proposed a multivariate estimator based on a weighted sum of single ratio and product estimators.

John (1969) suggested two alternative multivariate generalizations of ratio and product estimators which actually reduce to the Olkin's (1958) and Singh (1967) estimators. Srivastava (1971) considered a general ratio-type estimator which generates a large class of estimators including most of the estimators up to date.

Many other contributions are present in sampling literature and, recently, some new estimators appeared. Robinson (1994), suggest a regression estimator ignoring some of the assumptions usually adopted in the literature, Srivastava, (1971). Tracy et al. (1996) and Perri (2004), when two auxiliary variables are available, suggested an alternative to Singh $(1965,1967)$ ratio-cum-product estimators. Ceccon and Diana (1996) gave a multivariate extension of the Naik and Gupta (1991) univariate class of estimators. Agarwal et al. (1997), moving from Raj (1965), illustrated a new approach to form a multivariate difference estimator which does not require the knowledge of any population parameters. Abu-Dayyeh et al. (2003) introduced two estimators which are indeed members of the class proposed by Srivastava (1971), while Kadilar and Cingi $(2004,2005)$ analyzed combinations of regression type estimators in the case of two auxiliary variables. In the same situation, Perri (2005) proposed some new estimators obtained from Singh $(1965,1967)$ ones. 
Typically, almost all the estimators are based on the known means of auxiliary variables are nonlinear in the auxiliary means and the efficiency is studied through the first order approximation of their mean square error Wolter (1985). But, in spite of the wide variety of estimators, many estimators exhibit the same performance at the first order of approximation. Moreover, they generally do not consider other sources of supplementary information such as that one provided by the moments of order greater than the first. As a matter of fact, some works deal with the problem of estimating the unknown mean of a study variable by using the means and variances of the auxiliary variables, but only in a two-phase simple random sampling scheme, Diana and Tommasi (2003), for a review. The use of multi-auxiliary information about means and variances is scarcely considered outside the double sampling Das and Tripathi (1981) in the case of a single auxiliary variable.

In addition most of the authors either propose a new estimator or developed the existing estimator leaving the concept of comparison among the existing estimators silent. In this regard an attempt is made to compare two existing estimators to determine the most efficient.

\section{1 source Of Data}

\section{Materials And Methods}

The data used in this study were collected from Federal Inland Revenue Service (FIRS) Abuja. The data consist of 200 observations of overhead expenditure on office routine electronic equipment's and 200 observations of overhead expenditure on office routine furniture equipment's for the year 2011. The data is based on the cost of items $(\mathrm{Y})$, manufacture length $\left(X_{1}\right)$ and days in the store $\left(X_{2}\right)$. Large sample size $\mathrm{n}>30$ units will be selected.

\subsection{Olkin (1958) Conventional Multivariate Ratio Estimator}

Olkin (1958) presented the multivariate average ratio estimator using information of two auxiliary variables $x_{1}$ and $x_{2}$ to estimate the population mean $(\bar{Y})$, as follows:

$$
\bar{Y}_{M A V G}=w_{1} \bar{y} \frac{\bar{X}_{1}}{\bar{x}_{1}}+w_{2} \bar{y} \frac{\bar{X}_{2}}{\bar{x}_{2}}
$$

Where $\bar{y}$ denotes the sample mean of the variable of interest $\mathrm{y} ; x_{i}$ and $x_{i}(i=1,2)$ denote respectively the sample and the population means of the variable $x_{i}$; and $w_{1}, w_{2}$ are the weights that satisfy the condition: $w_{1}=w_{2}=\frac{1}{2}$. The Olkin estimator becomes;

$\bar{Y}_{M A V G}=\frac{1}{2} \bar{y}\left(\frac{\bar{X}_{1}}{\bar{x}_{1}}+\frac{\bar{X}_{2}}{\bar{x}_{2}}\right)(2.2 .2)$

The study shows the property of $\bar{y}$ as an estimator of $\bar{Y}$. Thus from 2.2.1 taking expectation;

$$
\begin{aligned}
& E\left(\bar{Y}_{M A V G}\right)=E\left(w_{1} \bar{y} \frac{\bar{X}_{1}}{\bar{x}_{1}}+w_{2} \bar{y} \frac{\bar{X}_{2}}{\bar{x}_{2}}\right) \\
& E\left(\bar{Y}_{M A V G}\right)=w_{1} \bar{y} E\left(\frac{\bar{X}_{1}}{\bar{x}_{1}}\right)+w_{2} \bar{y} E\left(\frac{\bar{X}_{2}}{\bar{x}_{2}}\right)
\end{aligned}
$$

Since $E(\bar{x})=\bar{X}$, then

$$
\begin{aligned}
& E\left(\bar{Y}_{M A V G}\right)=w_{1} \bar{y}\left(\frac{\bar{X}_{1}}{\bar{X}_{1}}\right)+w_{2} \bar{y}\left(\frac{\bar{X}_{2}}{\bar{X}_{2}}\right) \\
& E\left(\bar{Y}_{M A V G}\right)=w_{1} \bar{y}+w_{2} \bar{y} \\
& E\left(\bar{Y}_{M A V G}\right)=\bar{y}\left(w_{1}+w_{2}\right)
\end{aligned}
$$

But $w_{1}=w_{2}=\frac{1}{2}$

$E\left(\bar{Y}_{M A V G}\right)=\bar{y}$. This is unbiased

The MSE of this estimator is given by Singh (1986) as: 
$\operatorname{MSE}\left(\bar{Y}_{M A V G}\right)=\frac{1-f}{n} \bar{Y}^{2}\left\{C_{y}^{2}-2 w_{1} \rho_{y x_{1}} C_{y} C_{x_{1}}-2 w_{2} \rho_{y x_{2}} C_{y} C_{x_{2}}+w_{1} C_{x_{1}}^{2}+2 w_{1} w_{2} \rho_{x_{1} x_{2}} C_{x_{1}} C_{x_{2}}\right.$

$\left.+w_{2} C_{x_{2}}^{2}\right\}(2.2 .3)$

when $w_{1}=w_{2}=\frac{1}{2}(2.2 .3)$ becomes;

$\operatorname{MSE}\left(\bar{Y}_{M A V G}\right)=\frac{1-f}{4 n} \bar{Y}^{2}\left\{4 C_{y}^{2}-4 \rho_{y x_{1}} C_{y} C_{x_{1}}-4 \rho_{y x_{2}} C_{y} C_{x_{2}}+C_{x_{1}}^{2}+2 \rho_{x_{1} x_{2}} C_{x_{1}} C_{x_{2}}+C_{x_{2}}^{2}\right\}$

where $\mathrm{n}$ and $\mathrm{N}$ are the sample and the population sizes respectively; $f=\frac{n}{N}, C_{y}^{2}, C_{x_{1}}^{2}$ and $C_{x_{2}}^{2}$ denote the coefficient of variation of $\mathrm{Y}, X_{1}$ and $X_{2}$ respectively and $\rho_{y x_{1}}, \rho_{y x_{2}}, \rho_{x_{1} x_{2}}$ denote the correlation coefficient between $\mathrm{Y}$ and $X_{1}, \mathrm{Y}$ and $X_{2}, X_{1}$ and $X_{2}$ respectively, Abu-Dayyeh et al. (2003).

\subsection{The Kadilar And Cingi (2004) Estimator}

The Kadilar and Cingi Regression estimator, study used the following estimator:

$\bar{Y}_{R E G(p r)}=\tau_{1} \frac{\bar{y}+\beta_{1}\left(\bar{X}_{1}-\bar{x}_{1}\right)}{\bar{x}_{1}} \bar{X}_{1}+\tau_{2} \frac{\bar{y}+\beta_{2}\left(\bar{X}_{2}-\bar{x}_{2}\right)}{\bar{x}_{2}} \bar{X}_{2}$

Note

$\beta_{i \neq} 0,(i=1,2)$.

Where $\tau_{1}$ and $\tau_{2}$ are the weights that satisfy the condition: $\tau_{1}=\tau_{2}=\frac{1}{2} ; \beta_{1}$ and $\beta_{2}$ are the regression coefficients for $x_{1}$ and $x_{2}$ respectively.

The study shows the property of $\bar{y}$ as an estimator of $\bar{Y}$. Thus from 3.4.1 taking expectation;

$$
\begin{aligned}
& E\left(\bar{Y}_{R E G(p r)}\right)=E\left(\tau_{1} \frac{\bar{y}+\beta_{1}\left(\bar{X}_{1}-\bar{x}_{1}\right)}{\bar{x}_{1}} \bar{X}_{1}+\tau_{2} \frac{\bar{y}+\beta_{2}\left(\bar{X}_{2}-\bar{x}_{2}\right)}{\bar{x}_{2}} \bar{X}_{2}\right) \\
& E\left(\bar{Y}_{R E G(p r)}\right)=E\left(\frac{1}{x_{1}}\left\{\tau_{1} \bar{X}_{1} \bar{y}+\tau_{1} \bar{X}_{1}^{2} \beta_{1}-\tau_{1} \bar{X}_{1} \beta_{1} x_{1}\right\}\right)+E\left(\frac{1}{x_{2}}\left\{\tau_{2} \bar{X}_{2} \bar{y}+\tau_{2} \bar{X}_{2}^{2} \beta_{2}-\tau_{2} \bar{X}_{2} \beta_{2} x_{2}\right\}\right) \\
& E\left(\bar{Y}_{R E G(p r)}\right)=\tau_{1} \bar{y} E\left(\frac{\bar{X}_{1}}{\bar{x}_{1}}\right)+\tau_{1} \beta_{1} E\left(\frac{\bar{X}_{1}^{2}}{\bar{x}_{1}}\right)-\tau_{1} \beta_{1} E\left(\frac{\bar{X}_{1}}{\bar{x}_{1}} \bar{x}_{1}\right)+\tau_{2} \bar{y} E\left(\frac{\bar{X}_{2}}{\bar{x}_{2}}\right)+\tau_{2} \beta_{2} E\left(\frac{\bar{X}_{2}}{\bar{x}_{2}}\right)-\tau_{2} \beta_{2} E\left(\frac{\bar{X}_{2}}{\bar{x}_{2}} \bar{x}_{2}\right) \\
& E\left(\bar{Y}_{R E G(p r)}\right)=\tau_{1} \bar{y}+\tau_{1} \beta_{1} \bar{X}_{1}-\tau_{1} \beta_{1} \bar{X}_{1}+\tau_{2} \bar{y}+\tau_{2} \beta_{2} \bar{X}_{2}-\tau_{2} \beta_{2} \bar{X}_{2} \\
& E\left(\bar{Y}_{R E G(p r)}\right)=\tau_{1} \bar{y}+\tau_{2} \bar{y} \\
& E\left(\bar{Y}_{R E G(p r)}\right)=\bar{y}\left(\tau_{1}+\tau_{2}\right) \\
& \operatorname{But} \tau_{1}=\tau_{2}=\frac{1}{2} \\
& E\left(\bar{Y}_{R E G(p r)}\right)=\bar{y} \text {. This is also unbiased. }
\end{aligned}
$$

The MSE of this estimator can be found using Taylor series method defined by

$$
\operatorname{MSE}\left(\bar{Y}_{R E G(p r)}\right) \cong d \sum d^{T}
$$

where

$$
d=\left[\left.\left.\left.\frac{\partial\left(y, x_{1}, x_{2}\right)}{\partial y}\right|_{\bar{Y}, \bar{X}_{1}, \bar{X}_{2}} \quad \frac{\partial\left(y, x_{1}, x_{2}\right)}{\partial x_{1}}\right|_{\bar{Y}, \bar{X}_{1}, \bar{X}_{2}} \quad \frac{\partial\left(y, x_{1}, x_{2}\right)}{\partial x_{2}}\right|_{\bar{Y}, \bar{X}_{1}, \bar{X}_{2}}\right]
$$


$\Sigma=\frac{1-f}{n}\left[\begin{array}{ccc}S_{y}^{2} & S_{y x_{1}} & S_{y x_{2}} \\ S_{x_{1} y} & S_{x_{1}}^{2} & S_{x_{1} x_{2}} \\ S_{x_{2} y} & S_{x_{2} x_{1}} & S_{x_{2}}^{2}\end{array}\right]$

Here $S_{y}^{2}, S_{x_{1}}^{2}$ and $S_{x_{2}}^{2}$ denote the covariance between Yand $X_{1}$, Yand $X_{2}, X_{1}$ and $X_{2}$ respectively and $S_{y x_{1}}=S_{x_{1} y}, S_{y x_{2}}=S_{x_{2} y}, S_{x_{1} x_{2}}=S_{x_{2} x_{1}}$ denote the covariance between Yand $X_{1}$, Yand $X_{2}, X_{1}$ and $X_{2}$ respectively.

In order to determine the minimum attainable mean square error, we take the partial derivatives of $\left(\bar{Y}_{R E G(p r)}\right)$ with respect to components y, $x_{1}$ and $x_{2}$ respectively. (2.3.1) becomes;

$$
\begin{aligned}
& \bar{Y}_{R E G(p r)}=\frac{1}{x_{1}}\left\{\tau_{1} \bar{X}_{1} \bar{y}+\tau_{1} \bar{X}_{1}^{2} \beta_{1}-\tau_{1} \bar{X}_{1} \beta_{1} x_{1}\right\}+\frac{1}{x_{2}}\left\{\tau_{2} \bar{X}_{2} \bar{y}+\tau_{2} \bar{X}_{2}^{2} \beta_{2}-\tau_{2} \bar{X}_{2} \beta_{2} x_{2}\right\} \\
& \frac{\partial\left(y, x_{1}, x_{2}\right)}{\partial y}=\tau_{1} \frac{\bar{X}_{1}}{x_{1}}+\left.\tau_{2} \frac{\bar{X}_{2}}{x_{2}}\right|_{y=\bar{Y}, x_{1}=\bar{X}_{1}, x_{2}=\bar{X}_{2}} \\
& \quad=\tau_{1}+\tau_{2}=1 \\
& \frac{\partial\left(y, x_{1}, x_{2}\right)}{\partial x_{1}}=-\tau_{1} \frac{\bar{X}_{1}}{x_{1}^{2}} \bar{y}-\left.\tau_{1} \frac{\bar{X}_{1}^{2}}{x_{1}^{2}} \beta_{1}\right|_{y=\bar{Y}, x_{1}=\bar{X}_{1}, x_{2}=\bar{X}_{2}} \\
& =-\tau_{1}\left(\frac{\bar{Y}}{\bar{X}_{1}}+\beta_{1}\right) \\
& \frac{\partial\left(y, x_{1}, x_{2}\right)}{\partial x_{2}}=-\tau_{2} \frac{\bar{X}_{2}}{x_{2}^{2}} \bar{y}-\left.\tau_{2} \frac{\bar{X}_{2}^{2}}{x_{2}^{2}} \beta_{2}\right|_{y=\bar{Y}, x_{1}=\bar{X}_{1}, x_{2}=\bar{X}_{2}} \\
& =-\tau_{2}\left(\frac{\bar{Y}_{\bar{X}}}{\bar{X}_{2}}+\beta_{2}\right)
\end{aligned}
$$

According to this definition, the study obtained dfor the estimator as

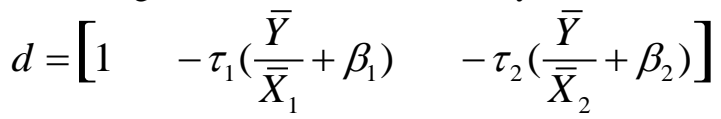

where $\beta_{1}=\frac{S_{y x_{1}}}{S_{x_{1}}^{2}}=\frac{\rho_{y x_{1}} S_{y} S_{x_{1}}}{S_{x_{1}}^{2}}$ and $\beta_{2}=\frac{S_{y x_{2}}}{S_{x_{2}}^{2}}=\frac{\rho_{y x_{2}} S_{y} S_{x_{2}}}{S_{x_{2}}^{2}}$

The study obtains MSE of the estimator using (2.3.2) as follows:

$$
\begin{aligned}
& \operatorname{MSE}\left(\bar{Y}_{R E G(p r)}\right)=\frac{1-f}{n}\left\{S_{y}^{2}+\tau_{1}^{2}\left(\frac{\bar{Y}}{\bar{X}_{1}}+\beta_{1}\right)^{2} S_{x_{1}}^{2}+\tau_{2}^{2}\left(\frac{\bar{Y}}{\bar{X}_{2}}+\beta_{2}\right)^{2} S_{x_{2}}^{2}-2 \tau_{1}\left(\frac{\bar{Y}}{\bar{X}_{1}}+\beta_{1}\right) S_{x_{1} y}\right. \\
& -2 \tau_{2}\left(\frac{\bar{Y}}{\bar{X}_{2}}+\beta_{2}\right) S_{x_{2} y}+2 \tau_{1} \tau_{2}\left(\frac{\bar{Y}}{\bar{X}_{1}}+\beta_{1}\right)\left(\frac{\bar{Y}}{\bar{X}_{2}}+\beta_{2}\right) S_{x_{1} x_{2}}
\end{aligned}
$$

We can also write (2.3.4) by

$$
\begin{aligned}
& \operatorname{MSE}\left(\bar{Y}_{R E G(p r)}\right)=\frac{1-f}{n} \bar{Y}^{2}\left\{C_{y}^{2}+\tau_{1}^{2}\left(C_{x_{1}}^{2}+2 C_{y x_{1}}+\rho_{y x_{1}}^{2} C_{y}^{2}\right)+\tau_{2}^{2}\left(C_{x_{2}}^{2}+2 C_{y x_{2}}+\rho_{y x_{2}}^{2} C_{y}^{2}\right)\right. \\
& -2 \tau_{1}\left(C_{y x_{1}}+\rho_{y x_{1}}^{2} C_{y}^{2}\right)-2 \tau_{2}\left(C_{y x_{2}}+\rho_{y x_{2}}^{2} C_{y}^{2}\right)+2 \tau_{1} \tau_{2}\left(C_{x_{1} x_{2}}+\rho_{y x_{2}} \rho_{x_{1} x_{2}} C_{x_{1}} C_{y}\right. \\
& \left.\left.+\rho_{y x_{1}} \rho_{x_{1} x_{2}} C_{x_{2}} C_{y}+\rho_{y x_{1}} \rho_{y x_{2}} \rho_{x_{1} x_{2}} C_{y}^{2}\right)\right\} \\
& \text { Where } C_{y x_{1}}=\frac{S_{y x_{1}}}{\overline{Y X}_{1}}, C_{y x_{2}}=\frac{S_{y x_{2}}}{\overline{Y X}_{2}} \text { and } C_{x_{1} x_{2}}=\frac{S_{x_{1} x_{2}}}{\bar{X}_{1} \bar{X}_{2}}
\end{aligned}
$$




\subsection{Comparison Of The Estimators}

This study compares the mean square error (MSE) of the Kadilar and Cingi (2004) estimator given in (2.3.5) with the Olkin (1958) estimator given in (3.2.4). The study has the conditions as follows:

$$
\operatorname{Efficiency}(E)=\frac{\operatorname{MSE}\left(\bar{Y}_{R E G(p r)}\right)}{\operatorname{MSE}\left(\bar{Y}_{M A V G}\right)}
$$

If:

(i) $\operatorname{MSE}\left(\bar{Y}_{R E G(p r)}\right)=\operatorname{MSE}\left(\bar{Y}_{M A V G}\right)$, then Efficiency $(\mathrm{E})=1$. Therefore, the two estimators are the same.

(ii) $\frac{\operatorname{MSE}\left(\bar{Y}_{R E G(p r)}\right)}{\operatorname{MSE}\left(\bar{Y}_{M A V G}\right)}<1$, then $\operatorname{MSE}\left(\bar{Y}_{R E G(p r)}\right)$ is smaller than $\operatorname{MSE}\left(\bar{Y}_{M A V G}\right)$. Therefore,

$\operatorname{MSE}\left(\bar{Y}_{R E G(p r)}\right)$ is more efficient than $\operatorname{MSE}\left(\bar{Y}_{M A V G}\right)$.

(iii) Otherwise, $\operatorname{MSE}\left(\bar{Y}_{M A V G}\right)$ is more efficient than $\operatorname{MSE}\left(\bar{Y}_{R E G(p r)}\right)$.

Now the Kadilar and Cingi (2004) estimator will be more efficient than the Olkin (1958) estimator if:

$\operatorname{MSE}\left(\bar{Y}_{M A V G}\right)-\operatorname{MSE}\left(\bar{Y}_{R E G(p r)}\right)<0$

Now using (3.2.4) and (3.3.5) we have:

$$
\begin{aligned}
& \operatorname{MSE}\left(\bar{Y}_{M A V G}\right)-\operatorname{MSE}\left(\bar{Y}_{R E G(p r)}\right)=3 C_{y}^{2}+C_{x_{1}}^{2}\left(1-\tau_{1}^{2}\right)+C_{x_{2}}^{2}\left(1-\tau_{2}^{2}\right)-4 \rho_{y x_{1}} C_{y} C_{x_{1}}-4 \rho_{y x_{2}} C_{y} C_{x_{2}} \\
& +2 \rho_{x_{1} x_{2}} C_{x_{1}} C_{x_{2}}-\tau_{1}^{2}\left(2 C_{y x_{1}}+\rho_{y x_{1}}^{2} C_{y}^{2}\right)-\tau_{2}^{2}\left(2 C_{y x_{2}}+\rho_{y x_{2}}^{2} C_{y}^{2}\right) \\
& +2 \tau_{1}\left(C_{y x_{1}}+\rho_{y x_{1}}^{2} C_{y}^{2}\right)+2 \tau_{2}\left(C_{y x_{2}}+\rho_{y x_{2}}^{2} C_{y}^{2}\right)-2 \tau_{1} \tau_{2}\left(C_{x_{1} x_{2}}\right. \\
& \left.+\rho_{y x_{2}} \rho_{x_{1} x_{2}} C_{x_{1}} C_{y}+\rho_{y x_{1}} \rho_{x_{1} x_{2}} C_{x_{2}} C_{y}+\rho_{y x_{1}} \rho_{y x_{2}} \rho_{x_{1} x_{2}} C_{y}^{2}\right)
\end{aligned}
$$

$<0$

When this condition is satisfied, the Kadilar and Cingi (2004) estimator will be more efficient than the Olkin (1958) estimator.

\subsection{Introduction}

\section{Results And Discussion}

In this chapter empirical study is conducted to see the performance of the Kadilar and Cingi (2004) estimator over the estimator proposed by Olkin (1958). The values of variance-covariance are used to calculate efficiency of the estimator for various values of $X_{1}$ and $X_{2}$.

The data used for population 1 is based on record on overhead expenditure of office routine electronic equipment's cost of items (y) and period of items from the date manufactured $\left(X_{1}\right)$ and period of items stayed in the store $\left(X_{2}\right)$ for the year 2011. Then the samples of size 110 were selected.

The data used for population 2 is based on record on overhead expenditure of office routine furniture equipment's cost of items (y) and period of items from the date manufactured $\left(X_{1}\right)$ and period of items stayed in the store $\left(X_{2}\right)$ for the year 2011. Then the samples of size 150 are drawn using simple random sampling method. 
Table 3.1: Summary of Estimatesfor Populations 1 and 2

\begin{tabular}{|c|c|c|}
\hline Estimators & Populationl & Population2 \\
\hline$N=200$ & $\mathrm{~N}=200$ & \\
\hline $\mathrm{n}=110$ & $\mathrm{n}=150$ & \\
\hline \multicolumn{3}{|c|}{$f=0.55 f=0.75$} \\
\hline$\overline{\bar{Y}}$ & 60569 & 78350 \\
\hline $\bar{X}_{1}$ & 21489 & 29744 \\
\hline $\bar{X}_{2}$ & 7342 & 9641 \\
\hline$S_{y}$ & 3592055.850 & 2775008.116 \\
\hline$S_{x_{p}}$ & 27778.280 & 25611.539 \\
\hline$S_{x_{3}}$ & 4857.950 & 5304.808 \\
\hline$C_{y}$ & 59.305 & 35.418 \\
\hline$C_{*}$ & 1.293 & 0.861 \\
\hline$C_{x_{3}}$ & 0.662 & 0.550 \\
\hline$\rho_{m_{m_{1}}}$ & 0.113 & 0.146 \\
\hline$\rho_{y_{z}}$ & 0.039 & 0.054 \\
\hline$\rho_{s, s_{2}}$ & 0.136 & 0.147 \\
\hline
\end{tabular}

Table 3.2: Computed MSE for the Two Estimators

\begin{tabular}{|lc|}
\hline$M S E\left(\bar{Y}_{M A V G}\right)$ & $M S E\left(\bar{Y}_{R E G(p r)}\right) \operatorname{MSE}\left(\bar{Y}_{M A V G}\right)-\operatorname{MSE}\left(\bar{Y}_{R E G(p r)}\right)$ \\
\hline Population1 & $5.2614 \times 10^{11} 2.2279 \times 10^{11}-1.3074$ \\
\hline Population2 & $1.2791 \times 10^{10} 0.8695 \times 10^{10}-6.0622$ \\
\hline
\end{tabular}

However, $\frac{\operatorname{MSE}\left(\bar{Y}_{R E G(p r)}\right)}{\operatorname{MSE}\left(\bar{Y}_{M A V G}\right)}=\frac{2.2279 X 10^{11}}{5.2614 X 10^{11}}=0.4234$

And

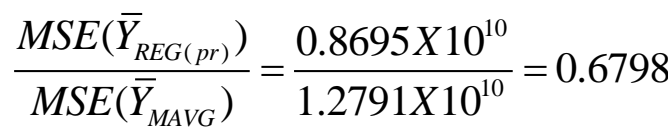

Table 3.2 shows efficiency comparison of the Kadilar and Cingi (2003) estimator with the Olkin (1958) estimator. The entries of table 3.2 clearly indicate that the Kadilar and Cingi (2004) estimator has smaller MSE than the Olkin (1958) estimator in the both populations. These results are not surprising because the value of the condition (2.3.6) are found as $<0$ and $\frac{\operatorname{MSE}\left(\bar{Y}_{R E G(p r)}\right)}{\operatorname{MSE}\left(\bar{Y}_{M A V G}\right)}<1$ therefore, $\operatorname{MSE}\left(\bar{Y}_{R E G(p r)}\right)$ is smaller than $\operatorname{MSE}\left(\bar{Y}_{M A V G}\right)$.

\section{Conclusion}

The study has found that Kandilar and Cingi (2004) multivariate ratio estimator has smaller mean square error than the Olkin (1958) average ratio estimator has. Therefore, the study concludes that the Kandilar and Cingi (2004) multivariate ratio estimator is more efficient than the Olkin (1958) average ratio estimator for this population.

\section{References}

[1]. Abu-Dayyeh, W. A., Ahmed, M. S., Ahmed, R. A., and Muttlak, H. A. (2003) Some Estimators of finite population mean using auxiliary information, Applied Mathematics and Computation, 139, 287-298.

[2]. Adewara, A. A., Job, O., Abidoye, A. O. and Oyeyemi' G' M'. (2008), Generalised Lynch Multivariate Estimate with K-parameters of Order $n^{-1}$.Journal of Mathematics Sciences Vol. 19 No. 2 187-191.

[3]. Agarwal, S. K., Sharma, U. K., and Kashyap, S. (1997) a new approach to use Multivariate auxiliary information in sample surveys, Journal of Statistical Planning and Inference, 60, 261-267.

[4]. Bahl, S. and Tuteja, R.K. (1991): Ratio and Product type exponential estimator, Information and Optimization sciences, Vol.XII, I, $159-163$.

[5]. Ceccon, C. and Diana, G. (1996) Classi di stimatori di tipo rapporto in presenza di píu variabiliausiliarie, Proceedings of Italian Statistical Society, XXXVIII Scientifi Meeting, Rimini, 9-13 April 1996, 119-126.

[6]. Cochran, W. G. (1942): Sampling theory when the sampling-units are of unequal sizes, Journal of American Statistics Association. 37, 199-212.

[7]. Cochran, W. G. (1977): Sampling Techniques, New York, John Wiley and Sons, $3^{\text {rd }}$ Edition. (ISBN. 0-471-16240-X. QA-276.6.C6, 001.4'222.77-728).

[8]. Kadilar, C. and Cingi, H. (2004) Estimator of a population mean using two auxiliary variables in simple random sampling, International Mathematical Journal, 5, 357-367.

[9]. Kadilar, C. and Cingi, H. (2005) A new estimator using two auxiliary variables, Applied Mathematics and Computation, 162, 901908.

[10]. Olkin, I. (1958) Multivariate ratio estimation for finite populations, Biometrika, 45, 154-165. 